\title{
An improved approximation lower bound for finding almost stable maximum matchings
}

\section{$\operatorname{AUTHOR}(S)$ :}

Hamada, Koki; Iwama, Kazuo; Miyazaki, Shuichi

\section{CITATION:}

Hamada, Koki ... [et al]. An improved approximation lower bound for finding almost stable maximum matchings. Information Processing Letters 2009, 109(18): 1036-1040

\section{ISSUE DATE:}

2009-08-31

URL:

http://hdl.handle.net/2433/226944

\section{RIGHT:}

(c) 2009. This manuscript version is made available under the CC-BY-NC-ND 4.0 license

http://creativecommons.org/licenses/by-nc-nd/4.0/; The full-text file will be made open to the public on 31 August 2011 in accordance with publisher's 'Terms and Conditions for Self-Archiving'; This is not the published version. Please cite only the published version.; この論文は出版社版でありません。引用の際には出版社版をご確認ご利用ください。 


\title{
An Improved Approximation Lower Bound for Finding Almost Stable Maximum Matchings
}

\author{
Koki Hamada ${ }^{\mathrm{a}}$, Kazuo Iwama ${ }^{\mathrm{a}}$, Shuichi Miyazaki ${ }^{\mathrm{b}}$ \\ ${ }^{a}$ Graduate School of Informatics, Kyoto University, Yoshida-Honmachi, Sakyo-ku, Kyoto 606-8501, \\ JAPAN \\ ${ }^{b}$ Academic Center for Computing and Media Studies, Kyoto University, Yoshida-Honmachi, Sakyo-ku, \\ Kyoto 606-8501, JAPAN
}

\begin{abstract}
In the stable marriage problem that allows incomplete preference lists, all stable matchings for a given instance have the same size. However, if we ignore the stability, there can be larger matchings. Biró et al. defined the problem of finding a maximum cardinality matching that contains minimum number of blocking pairs. They proved that this problem is not approximable within some constant $\delta>1$ unless $\mathrm{P}=\mathrm{NP}$, even when all preference lists are of length at most 3 . In this paper, we improve this constant $\delta$ to $n^{1-\varepsilon}$ for any $\varepsilon>0$, where $n$ is the number of men in an input.
\end{abstract}

Key words: approximation algorithms, the stable marriage problem, approximation ratio, polynomial-time reduction

\section{Introduction}

The stable marriage problem (SM for short), introduced by Gale and Shapley [3] (see also [7]), is defined as follows: An instance consists of the same number $n$ of men and women and each person's preference list. A preference list is a totally-ordered list that includes all members of the opposite sex in accordance with the owner's preference. The problem requires to find a stable matching, a perfect matching $M$ between men and women in which there is no pair of man $m$ and woman $w$, that are not matched together in $M$ but each prefers the other to one's partner in $M$. Such a pair $(m, w)$ is called a blocking pair for $M$. Gale and Shapley [3] proved that there is at least one stable matching in any instance, and proposed an $O\left(n^{2}\right)$-time algorithm to find one.

One possible extension of SM is to allow incomplete preference lists (SMI for short); namely, each person includes a subset of the members of the opposite sex in the preference list. Those who are included in a person $p$ 's preference list are said to be acceptable to $p$. Now a matching is defined as a set of disjoint pairs of mutually acceptable man and woman, and hence is not necessarily perfect. Accordingly, the definition of a blocking pair is extended as follows: A mutually acceptable pair of man $m$ and woman $w$ is a blocking pair for a matching $M$ if (i) $m$ and $w$ are not matched together in $M$, (ii) either

Email addresses: khamada@kuis.kyoto-u.ac.jp (Koki Hamada), iwama@kuis.kyoto-u.ac.jp (Kazuo Iwama), shuichi@media.kyoto-u.ac.jp (Shuichi Miyazaki) 
$m$ is single or prefers $w$ to his partner in $M$, and (iii) either $w$ is single or prefers $m$ to her partner in $M$. There can be many stable matchings for one instance, but all stable matchings are of the same size [6].

However, if we do not care about the stability, there can be larger matchings in general. So, we may sometimes want to obtain larger matchings by sacrificing the stability, but even in such a case, it is still natural to seek a matching which is as stable as possible. Related to this consideration, Biró et al. [2] defined the following optimization problem, called MAX SIZE MIN BP SMI: Given an SMI instance, find a matching that minimizes the number of blocking pairs among all the maximum cardinality matchings. For integers $p$ and $q$, MAX SIZE MIN BP $(p, q)$-SMI is the restriction of MAX SIZE MIN BP SMI so that each man's preference list is of length at most $p$, and each woman's preference list is of length at most $q . p=\infty$ or $q=\infty$ means that the lengths of preference lists are unbounded. Biró et al. [2] showed the following results; (1) MAX SIZE MIN BP $(\infty$, $\infty)$-SMI is NP-hard and cannot be approximated within the ratio of $n^{1-\varepsilon}$ for any constant $\varepsilon>0$, unless $\mathrm{P}=\mathrm{NP}$; (2) MAX SIZE MIN BP $(3,3)$-SMI is APX-hard and cannot be approximated within the ratio of $\frac{3557}{3556} \simeq 1.00028$ unless $\mathrm{P}=\mathrm{NP}$; (3) MAX SIZE MIN BP $(2, \infty)$-SMI is solvable in $O\left(n^{3}\right)$ time.

In this paper, we improve the hardness of the above (2), namely, we improve the constant $\frac{3557}{3556}$ to $n^{1-\varepsilon}$ for any constant $\varepsilon>0$. Our reduction uses basically the same idea as the one used in [2] to prove the above (1). In [2], some persons need to have preference lists of unbounded lengths for two reasons: One is for garbage collection, and the other is to create a large gap on the costs between "yes"-instances and "no"-instances. We perform a non-trivial modification of the construction and demonstrate that such gadgets can be replaced by persons with preference lists of length at most three.

Research on finding almost stable matchings is very active recently. Abraham et al. [1] consider the problem of finding a matching with the fewer blocking pairs in the stable roommates problem, and proved that in most variants the problem is hard, even to approximate. In addition to the number of blocking pairs, there are some other definitions of instability, such as the number of agents involved in blocking pairs and the number of blocking pairs relative to the size of the matching (see $[4,5]$ for example).

\section{Main Result}

Theorem 2.1. MAX SIZE MIN BP $(3,3)-S M I$ is not approximable within $n^{1-\varepsilon}$ where $n$ is the number of men in a given instance, for any $\varepsilon>0$, unless $P=N P$.

Proof. We demonstrate a polynomial-time reduction from the same problem as [2], EXACT Maximal Matching (EXACT-MM) restricted to subdivision graphs of cubic graphs, which is NP-complete [8]. A graph $G$ is a subdivision graph if it is obtained from another graph $H$ by replacing each edge $(u, v)$ of $H$ by two edges $(u, w)$ and $(w, v)$ where $w$ is a new vertex. In this problem, we are given a graph $G$ which is a subdivision graph of some cubic graph, as well as a positive integer $K$, and asked if $G$ contains a maximal matching of size exactly $K$. Hereafter, we simply say "EXACT-MM" to mean EXACT-MM with the above restrictions.

Given an instance $(G, K)$ of EXACT-MM, we construct an instance $I$ of MAX SIZE MIN BP (3,3)-SMI in such a way that (i) $I$ has a perfect matching, (ii) if $(G, K)$ is 
a "yes"-instance of EXACT-MM, then $I$ has a perfect matching with small number of blocking pairs, and (iii) if $(G, K)$ is a "no"-instance of EXACT-MM, then any perfect matching of $I$ has many blocking pairs.

$\left(\begin{array}{c}m \\ r\end{array}\right)$-gadget. Before going to the main body of the reduction, we first introduce the $\left(\begin{array}{c}m \\ r\end{array}\right)$ gadget. This gadget plays a role of garbage collection, just as $X$ and $Y$ in the proof of Theorem 1 of [2].

Let $X$ be a set of men of size $m$ where $X=\left\{x_{1}, \cdots, x_{m}\right\}$, and $r(0<r \leq m)$ be an integer. The $\left(\begin{array}{c}m \\ r\end{array}\right)$-gadget (with respect to $X$ and $r$ ), denoted $\mathcal{C}(X, r)$, consists of the following $2 m r-r$ men $\left(\bigcup_{1 \leq i \leq m} A_{i}\right) \cup\left(\bigcup_{1 \leq j \leq r} C_{j}\right)$ and $2 m r$ women $\left(\bigcup_{1 \leq i \leq m} B_{i}\right) \cup$ $\left(\bigcup_{1 \leq j \leq r} D_{j}\right)$.

$$
\begin{aligned}
& A_{i}=\left\{a_{i}^{j}: 1 \leq j \leq r\right\}, \quad B_{i}=\left\{b_{i}^{j}: 1 \leq j \leq r\right\} \quad(1 \leq i \leq m) \\
& C_{j}=\left\{c_{j}^{i}: 2 \leq i \leq m\right\}, \quad D_{j}=\left\{d_{j}^{i}: 1 \leq i \leq m\right\} \quad(1 \leq j \leq r)
\end{aligned}
$$

Each person's preference list is defined in Fig. 1. A person $p$ 's preference list " $p: a b c$ " means that $p$ prefers $a, b$, and $c$ in this order. For each $x_{i} \in X$, the unique woman $b_{i}^{1}$ of $\mathcal{C}(X, r)$ who includes $x_{i}$ in her preference list is referred to as $\mathcal{C}(X, r)\left[x_{i}\right]$.

$\begin{array}{llllllllll}a_{i}^{1} & : & d_{1}^{i} & b_{i}^{2} & b_{i}^{1} & b_{i}^{1} & : & a_{i}^{1} & x_{i} & \\ a_{i}^{2} & : & d_{2}^{i} & b_{i}^{3} & b_{i}^{2} & b_{i}^{2} & : & a_{i}^{2} & a_{i}^{1} & \\ a_{i}^{3} & : & d_{3}^{i} & b_{i}^{4} & b_{i}^{3} & b_{i}^{3} & : & a_{i}^{3} & a_{i}^{2} & \\ \vdots & & & & & \vdots & & & & \\ a_{i}^{r-1} & : & d_{r-1}^{i} & b_{i}^{r} & b_{i}^{r-1} & b_{i}^{r-1} & : & a_{i}^{r-1} & a_{i}^{r-2} & \\ a_{i}^{r} & : & d_{r}^{i} & b_{i}^{r} & & b_{i}^{r} & : & a_{i}^{r} & a_{i}^{r-1} & \\ & & & & d_{j}^{1} & : & c_{j}^{2} & a_{1}^{j} & \\ & & & d_{j}^{2} & : & c_{j}^{3} & c_{j}^{2} & a_{2}^{j} \\ c_{j}^{2} & : & d_{j}^{2} & d_{j}^{1} & d_{j}^{3} & : & c_{j}^{4} & c_{j}^{3} & a_{3}^{j} \\ c_{j}^{3} & : & d_{j}^{3} & d_{j}^{2} & d_{j}^{4} & : & c_{j}^{5} & c_{j}^{4} & a_{4}^{j} \\ c_{j}^{4} & : & d_{j}^{4} & d_{j}^{3} & \vdots & & & & \\ \vdots & & & & d_{j}^{m-1} & : & c_{j}^{m} & c_{j}^{m-1} & a_{m-1}^{j} \\ c_{j}^{m-1} & : & d_{j}^{m-1} & d_{j}^{m-2} & d_{j}^{m} & : & c_{j}^{m} & a_{m}^{j} & \\ c_{j}^{m} & : & d_{j}^{m} & d_{j}^{m-1} & & & & \end{array}$

Figure 1: Preference lists of $\mathcal{C}(X, r)$

The role of the gadget $\mathcal{C}(X, r)$ is to receive any subset $X^{\prime} \subseteq X$ such that $\left|X^{\prime}\right|=r$ without creating many blocking pairs, as formally stated in the following lemmas. In the following lemmas, we assume that each man $x_{i} \in X$ includes the woman $\mathcal{C}(X, r)\left[x_{i}\right]\left(=b_{i}^{1}\right)$ in his preference list.

Lemma 2.2. Let $X$ be a set of men and $r$ be an integer such that $0<r \leq|X|$. Then, for any $X^{\prime} \subseteq X$ such that $\left|X^{\prime}\right|=r$, there is a matching $M$ for $X$ and $\mathcal{C}(X, r)$ such that (i) all members of $\mathcal{C}(X, r)$ are matched, (ii) all men in $X^{\prime}$ are matched with women in $\mathcal{C}(X, r)$ 
and all men in $X \backslash X^{\prime}$ are single, and (iii) no person in $X$ is included in a blocking pair, and the number of blocking pairs for $M$ is at most $r$.

Proof. Let $X^{\prime}=\left\{x_{i_{1}}, x_{i_{2}}, \cdots, x_{i_{r}}\right\}\left(1 \leq i_{1}<i_{2}<\cdots<i_{r} \leq m\right)$. We construct the matching $M$ as follows. For each $j(1 \leq j \leq r)$, add the following pairs to $M:\left(a_{i_{j}}^{k}, b_{i_{j}}^{k+1}\right)$ for $k=1, \ldots, j-1 ;\left(a_{i_{j}}^{k}, b_{i_{j}}^{k}\right)$ for $k=j+1, \ldots, r ;\left(a_{i_{j}}^{j}, d_{j}^{i_{j}}\right) ;\left(c_{j}^{k+1}, d_{j}^{k}\right)$ for $k=1, \ldots, i_{j}-1$; $\left(c_{j}^{k}, d_{j}^{k}\right)$ for $k=i_{j}+1, \ldots, m$; and $\left(x_{i_{j}}, b_{i_{j}}^{1}\right)$. (Fig. 2 gives an example for a specific $i_{j}$.) Also, for each $i$ such that $x_{i} \in X \backslash X^{\prime}$, add $\left(a_{i}^{k}, b_{i}^{k}\right)$ for $k=1, \ldots, r$ to $M$. It is easy to see that (i) and (ii) are satisfied. Also, it is straightforward to check that blocking pairs are only $\left(c_{j}^{i_{j}}, d_{j}^{i_{j}}\right)\left(1 \leq j \leq r, i_{j} \neq 1\right)$, and hence there are at most $r$ blocking pairs.

Lemma 2.3. Let $X$ be a set of men and $r$ be an integer such that $0<r \leq|X|$. Let $M$ be any matching for $X$ and $\mathcal{C}(X, r)$ that matches all members of $\mathcal{C}(X, r)$. Then the number of single men in $X$ is $|X|-r$.

Proof. This is obvious because any member in $\mathcal{C}(X, r)$ includes only persons in $\mathcal{C}(X, r)$ and $X$ in the preference list, and there are $r$ more women than men in $\mathcal{C}(X, r)$.

When $X$ is a set of women, we similarly define the $\left(\begin{array}{c}m \\ r\end{array}\right)$-gadget by exchanging the roles of men and women.

Main Part of the Reduction. Let $I^{\prime}=(G, K)$ be an instance of EXACT-MM, where $G$ is a subdivision graph of some cubic graph and $K$ is a positive integer. Since $G$ is a bipartite graph, we can write it as $G=(U, W, E)$ such that $U=\left\{u_{1}, \cdots, u_{n_{1}}\right\}$ and $W=\left\{w_{1}, \cdots, w_{n_{2}}\right\}$, where each vertex in $U$ ( $W$, respectively) has degree exactly $2(3$, respectively). (Hence $n_{1}$ and $n_{2}$ are related as $2 n_{1}=3 n_{2}$.) Without loss of generality, we may assume that $K<\min (|U|,|W|)$ and that $G$ has a matching of size $K$.

As in [2], we give the following definitions: For each $u_{i} \in U$, let $w_{p_{i}}$ and $w_{q_{i}}$ be the two neighbors of $u_{i}$ in $G$, where $p_{i}<q_{i}$, and for each $w_{j} \in W$, let $u_{r_{j}}, u_{s_{j}}$, and $u_{t_{j}}$ be the three neighbors of $w_{j}$ in $G$, where $r_{j}<s_{j}<t_{j}$. Also, for each $u_{i} \in U$ and $w_{j} \in W$ such that $\left(u_{i}, w_{j}\right) \in E$, define $\sigma_{j, i}=1,2$ according to whether $w_{j}$ is $w_{p_{i}}$ or $w_{q_{i}}$ respectively, and define $\tau_{i, j}=1,2,3$ according to whether $u_{i}$ is $u_{r_{j}}$ or $u_{s_{j}}$ or $u_{t_{j}}$ respectively. For a given $\varepsilon>0$, define $B=\left\lceil\frac{3}{\varepsilon}\right\rceil$ and $C=\left(n_{1}+n_{2}\right)^{B+1}$.

For each vertex $u_{i} \in U$, we construct $2 C+3$ men and $2 C+2$ women, whose preference lists are given in Fig. 3, where men's lists are given in the left and women's lists are given in the right of the figure. We denote $\mathcal{U}\left(u_{i}\right)$ the set of these men and women. Define the set $U^{0}=\left\{u_{1}^{0}, \cdots, u_{n_{1}}^{0}\right\}$ (consisting of men, one from each $\left.\mathcal{U}\left(u_{i}\right)\left(1 \leq i \leq n_{1}\right)\right)$. We then construct $\left(\begin{array}{c}n_{1} \\ n_{1}-K\end{array}\right)$-gadget $\mathcal{C}\left(U^{0}, n_{1}-K\right)$.

Similarly, for each $w_{j} \in W$, we construct $3 C+3$ men and $3 C+4$ women, whose preference lists are given in Fig. 4 . We denote $\mathcal{W}\left(w_{j}\right)$ the set of these men and women. Define the set $W^{0}=\left\{w_{1}^{0}, \cdots, w_{n_{2}}^{0}\right\}$ (consisting of women, one from each $\mathcal{W}\left(w_{j}\right)(1 \leq j \leq$ $\left.n_{2}\right)$ ), and construct $\left(\begin{array}{c}n_{2} \\ n_{2}-K\end{array}\right)$-gadget $\mathcal{C}\left(W^{0}, n_{2}-K\right)$.

The reduction is now completed. The resulting instance $I$ contains the same number $n=\left(2+2 C+2 n_{1}-2 K\right) n_{1}+\left(3+3 C+2 n_{2}-2 K\right) n_{2}+K$ of men and women. Note that each person's preference list is of length at most three. It is not hard to see that the reduction can be performed in time polynomial in the size of $I^{\prime}$. 
Properties of Gadgets. Before proceeding to the correctness proof, we prove useful lemmas:

Lemma 2.4. For any edge $\left(u_{i}, w_{j}\right) \in E$, we can form a matching $M$ restricted to people in $\mathcal{U}\left(u_{i}\right) \cup \mathcal{W}\left(w_{j}\right)$ so that (i) all people in $\mathcal{U}\left(u_{i}\right) \cup \mathcal{W}\left(w_{j}\right)$ are matched, (ii) $M$ contains at most 2 blocking pairs, and (iii) for any extension of $M$ to a complete matching of $I$, no person in $\mathcal{U}\left(u_{i}\right) \cup \mathcal{W}\left(w_{j}\right)$ will create a blocking pair with a person not in $\mathcal{U}\left(u_{i}\right) \cup \mathcal{W}\left(w_{j}\right)$.

Proof. We construct a matching $M$ as follows. Since $\left(u_{i}, w_{j}\right) \in E$, there are integers $k$ and $l$ such that $\sigma_{j, i}=k$ and $\tau_{i, j}=l$, by the definition of $\sigma$ and $\tau$. We first add $\left(u_{i}^{k}, w_{j}^{l}\right)$ to $M$. Next, we consider people in $\mathcal{U}\left(u_{i}\right)$. Add the following pairs to $M:\left(g_{i, 1}^{1}, z_{i}^{2}\right) ;\left(g_{i, 1}^{s}, e_{i, 1}^{s-1}\right)$ for $s=2, \ldots, C ;\left(g_{i, 2}^{1}, e_{i, 1}^{C}\right) ;\left(g_{i, 2}^{s}, e_{i, 2}^{s-1}\right)$ for $s=2, \ldots, C$; and $\left(u_{i}^{0}, e_{i, 2}^{C}\right)$. If $k=1$, then add $\left(u_{i}^{2}, z_{i}^{1}\right)$, otherwise, add $\left(u_{i}^{1}, z_{i}^{1}\right)$. Finally, we consider people in $\mathcal{W}\left(w_{j}\right)$. Add the following pairs to $M:\left(v_{j}^{3}, h_{j, 1}^{1}\right) ;\left(f_{j, 1}^{s}, h_{j, 1}^{s+1}\right)$ for $s=1, \ldots, C-1 ;\left(f_{j, 1}^{C}, h_{j, 2}^{1}\right) ;\left(f_{j, 2}^{s}, h_{j, 2}^{s+1}\right)$ for $s=1, \ldots, C-1 ;\left(f_{j, 2}^{C}, h_{j, 3}^{1}\right) ;\left(f_{j, 3}^{s}, h_{j, 3}^{s+1}\right)$ for $s=1, \ldots, C-1$; and $\left(f_{j, 3}^{C}, w_{j}^{0}\right)$. If $l=1$, add $\left(v_{j}^{1}, w_{j}^{2}\right)$ and $\left(v_{j}^{2}, w_{j}^{3}\right)$; if $l=2$, add $\left(v_{j}^{1}, w_{j}^{1}\right)$ and $\left(v_{j}^{2}, w_{j}^{3}\right)$; if $l=3$, add $\left(v_{j}^{1}, w_{j}^{1}\right)$ and $\left(v_{j}^{2}, w_{j}^{2}\right)$.

It is straightforward to verify that Condition (i) is satisfied. To see that Conditions (ii) and (iii) hold, observe the following: $\operatorname{In} \mathcal{U}\left(u_{i}\right)$, all men of the form $g_{i, t}^{s}$ for any $t, s$, and $u_{i}^{0}$ are matched with their first choices. Clearly, these men do not form a blocking pair. Also, women who include only these men in their preference lists cannot form a blocking pair. So, only $u_{i}^{1}, u_{i}^{2}, z_{i}^{1}$, and $z_{i}^{2}$ can form a blocking pair. If we check the cases of $k=1$ and $k=2$, we can verify that at most one blocking pair is possible. Similarly, in $\mathcal{W}\left(w_{j}\right)$, all women of the form $h_{j, t}^{s}$ for any $t, s$, and $w_{j}^{0}$ are matched with their first choices. So, only $v_{j}^{1}, v_{j}^{2}, v_{j}^{3}, w_{j}^{1}, w_{j}^{2}$, and $w_{j}^{3}$ can be a part of a blocking pair. We may conclude that there is at most one blocking pair by checking cases $l=1,2,3$.

Lemma 2.5. In any matching of I that matches all members of $\mathcal{U}\left(u_{i}\right)\left(\mathcal{W}\left(w_{j}\right)\right.$, respectively), all people in $\mathcal{U}\left(u_{i}\right)\left(\mathcal{W}\left(w_{j}\right)\right.$, respectively), except for one man (woman, respectively), are matched among themselves.

Proof. This is true because any woman in $\mathcal{U}\left(u_{i}\right)$ includes only men in $\mathcal{U}\left(u_{i}\right)$ in her preference list. The case for $\mathcal{W}\left(w_{j}\right)$ can be proved similarly.

Lemma 2.6. Suppose that $\left(u_{i}, w_{j}\right) \in E$. Let $M$ be any matching of $I$ such that all people in $\mathcal{U}\left(u_{i}\right)$ and $\mathcal{W}\left(w_{j}\right)$ are matched by $M$ and both $\left(u_{i}^{0}, \mathcal{C}\left(U^{0}, n_{1}-K\right)\left[u_{i}^{0}\right]\right)$ and $\left(w_{j}^{0}, \mathcal{C}\left(W^{0}, n_{2}-K\right)\left[w_{j}^{0}\right]\right)$ are in $M$. Then there are at least $C$ blocking pairs for $M$ (formed by only people in $\left.\mathcal{U}\left(u_{i}\right) \cup \mathcal{W}\left(w_{j}\right)\right)$.

Proof. Since $\left(u_{i}^{0}, \mathcal{C}\left(U^{0}, n_{1}-K\right)\left[u_{i}^{0}\right]\right) \in M$ and all people in $\mathcal{U}\left(u_{i}\right)$ are matched in $M$, by tracing the women's preference lists, the partners of women in $\mathcal{U}\left(u_{i}\right)$ are uniquely determined, namely, $\left(g_{i, t}^{s}, e_{i, t}^{s}\right) \in M$ for any $t, s$, and $\left(u_{i}^{t}, z_{i}^{t}\right) \in M$ for $t=1,2$. Similarly, we may uniquely determine the pairs within $\mathcal{W}\left(w_{j}\right)$, namely, $\left(f_{j, t}^{s}, h_{j, t}^{s}\right) \in M$ for any $t, s$, and $\left(v_{j}^{t}, w_{j}^{t}\right) \in M$ for $t=1,2,3$.

Since $\left(u_{i}, w_{j}\right) \in E$, there are integers $k$ and $l$ such that $\sigma_{j, i}=k$ and $\tau_{i, j}=l$ by the definition of $\sigma$ and $\tau$. Then, all $\left(g_{i, k}^{s}, h_{j, l}^{s}\right)(1 \leq s \leq C)$ are blocking pairs for $M$. 
Correctness of the Reduction. We first show that $I$ admits a perfect matching. As we have assumed that $G$ has a matching of size $K$, let it be $M^{\prime}$. For each edge $\left(u_{i}, w_{j}\right) \in M^{\prime}$, we match people in $\mathcal{U}\left(u_{i}\right)$ and $\mathcal{W}\left(w_{j}\right)$ as in the proof of Lemma 2.4. There are exactly $n_{1}-K$ unmatched vertices in $U$. Let $\tilde{U}^{0}\left(\subseteq U^{0}\right)$ consist of men corresponding to these unmatched vertices, i.e. $\tilde{U}^{0}=\left\{u_{i}^{0}: u_{i} \in U\right.$ is unmatched in $\left.M^{\prime}\right\}$. We match people in $\tilde{U}^{0}$ and $\left(\begin{array}{c}n_{1} \\ n_{1}-K\end{array}\right)$-gadget $\mathcal{C}\left(U^{0}, n_{1}-K\right)$ as in the proof of Lemma 2.2. Also, for each $i$ such that $u_{i}^{0} \in \tilde{U}^{0}$, match every woman in $\mathcal{U}\left(u_{i}\right)$ to her first choice man. Similarly, there are exactly $n_{2}-K$ unmatched vertices in $W$. Define $\tilde{W}^{0}\left(\subseteq W^{0}\right)$ as $\tilde{W}^{0}=\left\{w_{j}^{0}\right.$ : $w_{j} \in W$ is unmatched in $\left.M^{\prime}\right\}$. Again, using the proof of Lemma 2.2, we match people in $\tilde{W}^{0}$ and $\left(\begin{array}{c}n_{2} \\ n_{2}-K\end{array}\right)$-gadget $\mathcal{C}\left(W^{0}, n_{2}-K\right)$. Finally, for each $j$ such that $w_{j}^{0} \in \tilde{W}^{0}$, match every man in $\mathcal{W}\left(w_{j}\right)$ to his first choice woman. By a careful observation, together with Lemma 2.2 (i) and (ii) and Lemma 2.4 (i), it can be verified that the above constriction yields a perfect matching.

Now suppose that $G$ has a maximal matching $M^{\prime}$ of size $K$. We construct a perfect matching $M$ of $I$ from $M^{\prime}$ as described above. We will count the number of blocking pairs for $M$. By Lemma 2.2 (iii), $\mathcal{C}\left(U^{0}, n_{1}-K\right)$ and $\mathcal{C}\left(W^{0}, n_{2}-K\right)$ contain at most $n_{1}-K$ and $n_{2}-K$ blocking pairs, respectively, and people in these gadgets do not create blocking pairs with people outside respective gadgets. Next we look at gadgets corresponding to vertices. For a pair of vertices $u_{i}$ and $w_{j}$ such that $\left(u_{i}, w_{j}\right) \in M^{\prime}$, there are at most 2 blocking pairs formed by people in $\mathcal{U}\left(u_{i}\right)$ and $\mathcal{W}\left(w_{j}\right)$ by Lemma 2.4 (ii). Since $\left|M^{\prime}\right|=K$, there are at most $2 K$ such blocking pairs. Also, by Lemma 2.4 (iii), people in $\mathcal{U}\left(u_{i}\right)$ and $\mathcal{W}\left(w_{j}\right)$ do not form blocking pairs with people outside $\mathcal{U}\left(u_{i}\right) \cup \mathcal{W}\left(w_{j}\right)$. Finally, we consider the gadgets corresponding to the vertices unmatched in $M^{\prime}$. Consider the gadget $\mathcal{U}\left(u_{i}\right)$ where $u_{i}$ is unmatched in $M^{\prime}$. By the construction of $M$, all women in $\mathcal{U}\left(u_{i}\right)$ are matched with their first choices, and cannot form a blocking pair. Hence only the possibility is that a man $g_{i, \sigma_{j, i}}^{s}$ forms a blocking pair with a woman $h_{j, \tau_{i, j}}^{s}$ for some $j$ and $s$. If this is the case, then $\left(u_{i}, w_{j}\right) \in E$, but by the maximality of $M^{\prime}, w_{j}$ is matched in $M^{\prime}$. Then, by the construction of $M, h_{j, \tau_{i, j}}^{s}$ must be matched with her first choice and hence $\left(g_{i, \sigma_{j, i}}^{s}, h_{j, \tau_{i, j}}^{s}\right)$ cannot be a blocking pair. Similarly, no people in $\mathcal{W}\left(w_{j}\right)$ where $w_{j}$ is unmatched in $M^{\prime}$ cannot form a blocking pair. In summary, the total number of blocking pairs is at most $\left(n_{1}-K\right)+\left(n_{2}-K\right)+2 K=n_{1}+n_{2}$.

Conversely, suppose that there is a perfect matching $M$ of $I$ that contains less than $C$ blocking pairs. By Lemma 2.5, for each $u_{i} \in U$, all people in $\mathcal{U}\left(u_{i}\right)$, except for one man (which we call a free-man), are matched among themselves. Hence there are exactly $n_{1}$ free-men. By Lemma 2.3, $M$ matches exactly $n_{1}-K$ men from $U^{0}$ with women in $\mathcal{C}\left(U^{0}, n_{1}-K\right)$. Clearly, all these men are free-men. So, there are $K$ remaining free-men. We will define free-women similarly, and by a similar argument, there are $K$ remaining free-women. Since $M$ is a perfect matching, these men and women are matched together.

Define $M^{\prime}$ as $M^{\prime}=\left\{\left(u_{i}, w_{j}\right):(x, y) \in M, x \in \mathcal{U}\left(u_{i}\right), y \in \mathcal{W}\left(w_{j}\right)\right\}$. If $(x, y) \in M$ for some $x\left(\in \mathcal{U}\left(u_{i}\right)\right)$ and $y\left(\in \mathcal{W}\left(w_{j}\right)\right)$, then $\left(u_{i}, w_{j}\right) \in E$ by the construction of preference lists of $I$. Also, it is easy to see that $x$ and $y$ are one of $K$ free-men and free-women, respectively, mentioned above. Hence, $M^{\prime}$ is a matching of $G$ of size $K$. We show that $M^{\prime}$ is maximal. For, suppose not. Then, there is an edge $\left(u_{i}, w_{j}\right) \in E$ both of whose endpoints are unmatched in $M^{\prime}$. By the construction of $M^{\prime}, u_{i}^{0} \in \mathcal{U}\left(u_{i}\right)$ is matched with the woman 
$\mathcal{C}\left(U^{0}, n_{1}-K\right)\left[u_{i}^{0}\right]$ and $w_{j}^{0} \in \mathcal{W}\left(w_{j}\right)$ is matched with the man $\mathcal{C}\left(W^{0}, n_{2}-K\right)\left[w_{j}^{0}\right]$, in $M$. But then by Lemma 2.6, $M$ contains at least $C$ blocking pairs, a contradiction. Hence $M^{\prime}$ is maximal, and we can conclude that if $G$ has no maximal matching of size $K$, then there is no perfect matching of $I$ with less than $C\left(=\left(n_{1}+n_{2}\right)^{B+1}\right)$ blocking pairs.

Hence, the existence of $\left(n_{1}+n_{2}\right)^{B}$-approximation algorithm for MAX SIZE MIN BP $(3,3)$-SMI implies a polynomial-time algorithm for EXACT-MM, which implies $\mathrm{P}=\mathrm{NP}$. We will show that $\left(n_{1}+n_{2}\right)^{B} \geq n^{1-\varepsilon}$. Recall that

$$
n=\left(2+2 C+2 n_{1}-2 K\right) n_{1}+\left(3+3 C+2 n_{2}-2 K\right) n_{2}+K,
$$

by which we obtain $n \leq 5\left(n_{1}+n_{2}\right)^{B+2}$, and hence

$$
\left(n_{1}+n_{2}\right)^{B} \geq 5^{-\frac{B}{B+2}} n^{\frac{B}{B+2}} .
$$

We may assume without loss of generality that $n_{1} \geq 3$. Since each vertex in $U$ and $W$ has degree 2 and 3 , respectively, $2 n_{1}=3 n_{2}$. So, we have $n_{1}+n_{2} \geq 5$. Also, $K<\min \left(n_{1}, n_{2}\right)$ by hypothesis. Thus, Equation (1) implies that $n \geq 5^{B}$ and hence, $5^{-\frac{B}{B+2}} \geq n^{-\frac{1}{B+2}}$. Since $B+2 \geq \frac{3}{\varepsilon}$, Inequality (2) implies that $\left(n_{1}+n_{2}\right)^{B} \geq n^{1-\varepsilon}$, which completes the proof.

\section{References}

[1] D. J. Abraham, P. Biró and D. F. Manlove, " "Almost stable" matchings in the roommates problem," Proc. WAOA 2005, LNCS 3879, pp. 1-14, 2005.

[2] P. Biró, D. F. Manlove, and S. Mittal, "Size versus stability in the marriage problem," Proc. WAOA 2008, LNCS 5426, pp. 15-28, 2008 (Full version: Technical Report TR2008-283, University of Glasgow, Department of Computing Science, 2008).

[3] D. Gale and L. S. Shapley, "College admissions and the stability of marriage," Amer. Math. Monthly, Vol. 69, pp. 9-15, 1962.

[4] K. Eriksson and O. Häggström, "Instability of matchings in decentralized markets with various preference structures," International Journal of Game Theory, Vol. 36, Issue 3, pp. 409-420, 2008.

[5] P. Floréen, P. Kaski, V. Polishchuk, and J. Suomela, "Almost stable matchings in constant time," CoRR abs/0812.4893, 2008.

[6] D. Gale and M. Sotomayor, "Some remarks on the stable matching problem," Discrete Applied Mathematics, Vol. 11, pp. 223-232, 1985.

[7] D. Gusfield and R. W. Irving, "The Stable Marriage Problem: Structure and Algorithms," MIT Press, Boston, MA, 1989.

[8] G. O'Malley, "Algorithmic aspects of stable matching problems," PhD thesis, University of Glasgow, 2007. 


$$
\begin{aligned}
& a_{i_{j}}^{1}: d_{1}^{i_{j}} \quad b_{i_{j}}^{2} \quad b_{i_{j}}^{1} \quad b_{i_{j}}^{1}: a_{i_{j}}^{1} x_{i_{j}}
\end{aligned}
$$

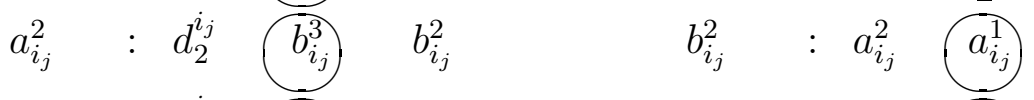

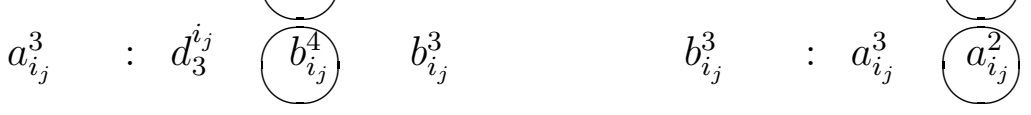

$$
\begin{aligned}
& \vdots
\end{aligned}
$$

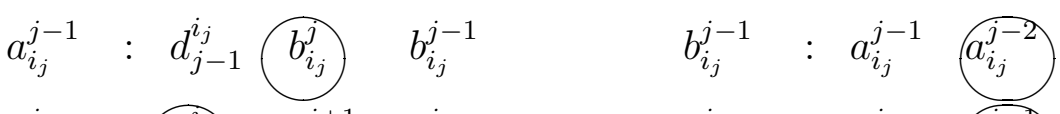

$$
\begin{aligned}
& a_{i_{j}}^{j}: d_{j}^{i_{j}} b_{i_{j}}^{j+1} b_{i_{j}}^{j} \quad b_{i_{j}}^{j}: a_{i_{j}}^{j} \quad \widehat{a_{i_{j}}^{j-1}} \\
& a_{i_{j}}^{j+1}: d_{j+1}^{i_{j}} b_{i_{j}}^{j+2} \quad b_{i_{j}}^{j+1} \quad: a_{i_{j}}^{j+1} a_{i_{j}}^{j}
\end{aligned}
$$

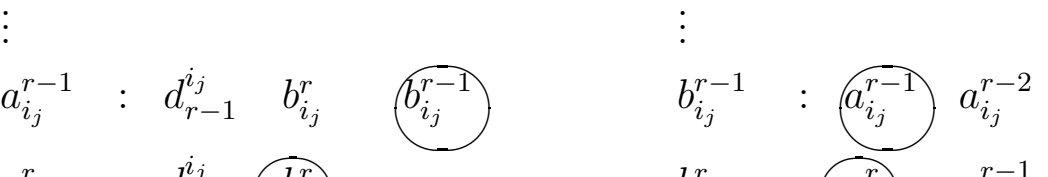

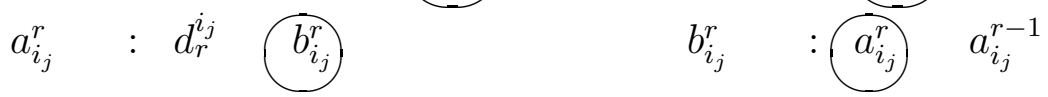

$$
\begin{aligned}
& d_{j}^{1} \quad: c_{j}^{2} \quad a_{1}^{j} \\
& \begin{array}{c:ccccc}
c_{j}^{2} & : d_{j}^{2} \quad d_{j}^{1} & d_{j}^{2} & c_{j}^{2} & a_{2}^{j}
\end{array} \\
& c_{j}^{3}: d_{j}^{3} \quad d_{j}^{2}: c_{j}^{4} c_{j}^{3} a_{3}^{j} \\
& c_{j}^{4}: d_{j}^{4} \quad d_{j}^{3}: c_{j}^{5} c_{j}^{4} a_{4}^{j}
\end{aligned}
$$

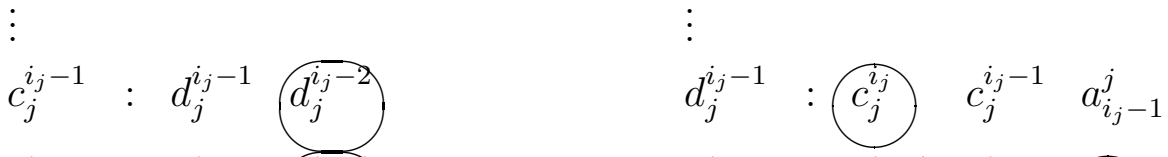

$$
\begin{aligned}
& c_{j}^{i_{j}}: d_{j}^{i_{j}} \quad d_{j}^{i_{j}-1} \quad: c_{j}^{i_{j}+1} c_{j}^{i_{j}} a_{i_{j}}^{j} \\
& c_{j}^{i_{j}+1}: d_{j}^{i_{j}+1} d_{j}^{i_{j}} \quad d_{j}^{i_{j}+1}: c_{j}^{i_{j}+2} c_{j}^{i_{j}+1} a_{i_{j}+1}^{j} \\
& \begin{array}{llll}
c_{j}^{m-1} & : d_{j}^{m-1} d_{j}^{m-2} & d_{j}^{m-1} & : c_{j}^{m} \\
c_{j}^{m} & : d_{j}^{m-1} d_{j}^{m-1} & d_{j}^{m} & : c_{j}^{m} a_{m}^{j}
\end{array}
\end{aligned}
$$

Figure 2: A part of the matching described in the proof of Lemma 2.2 


\begin{tabular}{|c|c|c|c|c|c|c|}
\hline$u_{i}^{1}$ & $: z_{i}^{1}$ & $w_{p_{i}}^{\tau_{i, p_{i}}}$ & & $z_{i}^{1}$ & $: \quad u_{i}^{1}$ & $u_{i}^{2}$ \\
\hline$u_{i}^{2}$ & $: \quad z_{i}^{1}$ & $z_{i}^{2}$ & $w_{q_{i}}^{\tau_{i, q_{i}}}$ & $z_{i}^{2}$ & $: u_{i}^{2}$ & $g_{i, 1}^{1}$ \\
\hline$g_{i, 1}^{1}$ & $: z_{i}^{2}$ & $h_{p_{i}, \tau_{i, p_{i}}}^{1}$ & $e_{i, 1}^{1}$ & $e_{i, 1}^{1}$ & $: \quad g_{i, 1}^{1}$ & $g_{i, 1}^{2}$ \\
\hline$g_{i, 1}^{2}$ & $: \quad e_{i, 1}^{1}$ & $h_{p_{i}, \tau_{i, p_{i}}}^{2}$ & $e_{i, 1}^{2}$ & $e_{i, 1}^{2}$ & $: g_{i, 1}^{2}$ & $g_{i, 1}^{3}$ \\
\hline$g_{i, 1}^{3}$ & $: \quad e_{i, 1}^{2}$ & $h_{p_{i}, \tau_{i, p_{i}}}^{3}$ & $e_{i, 1}^{3}$ & $e_{i, 1}^{3}$ & $: \quad g_{i, 1}^{3}$ & $g_{i, 1}^{4}$ \\
\hline & & & & $\vdots$ & & \\
\hline$g_{i, 1}^{C-1}$ & $: e_{i, 1}^{C-2}$ & $h_{p_{i}, \tau_{i, p_{i}}}^{C-1}$ & $e_{i, 1}^{C-1}$ & $e_{i, 1}^{C-1}$ & $: \quad g_{i, 1}^{C-1}$ & $g_{i, 1}^{C}$ \\
\hline$g_{i, 1}^{C}$ & $: e_{i, 1}^{C-1}$ & $h_{p_{i}, \tau_{i, p_{i}}}^{C}$ & $e_{i, 1}^{C}$ & $e_{i, 1}^{C}$ & $: \quad g_{i, 1}^{C}$ & $g_{i, 2}^{1}$ \\
\hline$g_{i, 2}^{1}$ & $: e_{i, 1}^{C}$ & $h_{q_{i}, \tau_{i, q_{i}}}^{1}$ & $e_{i, 2}^{1}$ & $e_{i, 2}^{1}$ & $: \quad g_{i, 2}^{1}$ & $g_{i, 2}^{2}$ \\
\hline$g_{i, 2}^{2}$ & $: \quad e_{i, 2}^{1}$ & $h_{q_{i}, \tau_{i, q_{i}}}^{2}$ & $e_{i, 2}^{2}$ & $e_{i, 2}^{2}$ & $: g_{i, 2}^{2}$ & $g_{i, 2}^{3}$ \\
\hline$g_{i, 2}^{3}$ & $: \quad e_{i, 2}^{2}$ & $h_{q_{i}, \tau_{i, q_{i}}}^{3}$ & $e_{i, 2}^{3}$ & $e_{i, 2}^{3}$ & $: \quad g_{i, 2}^{3}$ & $g_{i, 2}^{4}$ \\
\hline$\vdots$ & & & & $\vdots$ & & \\
\hline$g_{i, 2}^{C-1}$ & $: e_{i, 2}^{C-2}$ & $h_{q_{i}, \tau_{i, q_{i}}}^{C-1}$ & $e_{i, 2}^{C-1}$ & $e_{i, 2}^{C-1}$ & $: g_{i, 2}^{C-1}$ & $g_{i, 2}^{C}$ \\
\hline$g_{i, 2}^{C}$ & $: e_{i, 2}^{C-1}$ & $h_{q_{i}, \tau_{i, q_{i}}}^{C}$ & $e_{i, 2}^{C}$ & $e_{i, 2}^{C}$ & $: g_{i, 2}^{C}$ & $u_{i}^{0}$ \\
\hline$u_{i}^{0}$ & $: e_{i, 2}^{C}$ & $\mathcal{C}\left(U^{0}, n\right.$ & $1-K)\left[u_{i}^{0}\right]$ & & & \\
\hline
\end{tabular}

Figure 3: Preference lists of $\mathcal{U}\left(u_{i}\right)$ 


$$
\begin{array}{llll}
v_{j}^{1} & : & w_{j}^{1} & w_{j}^{2} \\
v_{j}^{2} & : & w_{j}^{2} & w_{j}^{3} \\
v_{j}^{3} & : & w_{j}^{3} & h_{j, 1}^{1} \\
f_{j, 1}^{1} & : & h_{j, 1}^{1} & h_{j, 1}^{2} \\
f_{j, 1}^{2} & : & h_{j, 1}^{2} & h_{j, 1}^{3} \\
f_{j, 1}^{3} & : & h_{j, 1}^{3} & h_{j, 1}^{4} \\
\vdots & & & \\
f_{j, 1}^{C-1} & : & h_{j, 1}^{C-1} & h_{j, 1}^{C} \\
f_{j, 1}^{C} & : & h_{j, 1}^{C} & h_{j, 2}^{1} \\
f_{j, 2}^{1} & : & h_{j, 2}^{1} & h_{j, 2}^{2} \\
f_{j, 2}^{2} & : & h_{j, 2}^{2} & h_{j, 2}^{3} \\
f_{j, 2}^{3} & : & h_{j, 2}^{3} & h_{j, 2}^{4} \\
\vdots & & & \\
f_{j, 2}^{C-1} & : & h_{j, 2}^{C-1} & h_{j, 2}^{C} \\
f_{j, 2}^{C} & : & h_{j, 2}^{C} & h_{j, 3}^{1} \\
f_{j, 3}^{1} & : & h_{j, 3}^{1} & h_{j, 3}^{2} \\
f_{j, 3}^{2} & : & h_{j, 3}^{2} & h_{j, 3}^{3} \\
f_{j, 3}^{3} & : & h_{j, 3}^{3} & h_{j, 3}^{4} \\
\vdots & & & \\
f_{j, 3}^{C-1} & : & h_{j, 3}^{C-1} & h_{j, 3}^{C} \\
f_{j, 3}^{C} & : & h_{j, 3}^{C} & w_{j}^{0}
\end{array}
$$$$
w_{j}^{1} \quad: v_{j}^{1} \quad u_{r_{j}}^{\sigma_{j, r_{j}}}
$$$$
w_{j}^{2} \quad: v_{j}^{1}
$$$$
v_{j}^{2} \quad u_{s_{j}}^{\sigma_{j, s_{j}}}
$$$$
w_{j}^{3} \quad: v_{j}^{2}
$$$$
v_{j}^{3}
$$$$
u_{t_{j}}^{\sigma_{j, t_{j}}}
$$$$
h_{j, 1}^{1} \quad: v_{j}^{3}
$$$$
g_{r_{j}, \sigma_{j, r_{j}}}^{1} f_{j, 1}^{1}
$$$$
h_{j, 1}^{2} \quad: \quad f_{j, 1}^{1} \quad g_{r_{j}, \sigma_{j, r_{j}}}^{2} f_{j, 1}^{2}
$$$$
h_{j, 1}^{3} \quad: \quad f_{j, 1}^{2} \quad g_{r_{j}, \sigma_{j, r_{j}}}^{3} f_{j, 1}^{3}
$$$$
h_{j, 1}^{C-1}: f_{j, 1}^{C-2} \quad g_{r_{j}, \sigma_{j, r_{j}}}^{C-1} f_{j, 1}^{C-1}
$$$$
h_{j, 1}^{C} \quad: \quad f_{j, 1}^{C-1} \quad g_{r_{j}, \sigma_{j, r_{j}}}^{C} f_{j, 1}^{C}
$$$$
h_{j, 2}^{1} \quad: f_{j, 1}^{C} \quad g_{s_{j}, \sigma_{j, s_{j}}}^{1} f_{j, 2}^{1}
$$$$
h_{j, 2}^{2} \quad: \quad f_{j, 2}^{1} \quad g_{s_{j}, \sigma_{j, s_{j}}}^{2} f_{j, 2}^{2}
$$$$
h_{j, 2}^{3} \quad: \quad f_{j, 2}^{2} \quad g_{s_{j}, \sigma_{j, s_{j}}}^{3} f_{j, 2}^{3}
$$$$
h_{j, 2}^{C-1}: f_{j, 2}^{C-2} \quad g_{s_{j}, \sigma_{j, s_{j}}}^{C-1} f_{j, 2}^{C-1}
$$$$
h_{j, 2}^{C} \quad: \quad f_{j, 2}^{C-1} \quad g_{s_{j}, \sigma_{j, s_{j}}}^{C} f_{j, 2}^{C}
$$$$
h_{j, 3}^{1} \quad: \quad f_{j, 2}^{C} \quad g_{t_{j}, \sigma_{j, t_{j}}}^{1} \quad f_{j, 3}^{1}
$$$$
h_{j, 3}^{2} \quad: \quad f_{j, 3}^{1} \quad g_{t_{j}, \sigma_{j, t_{j}}}^{2} \quad f_{j, 3}^{2}
$$$$
h_{j, 3}^{3} \quad: \quad f_{j, 3}^{2} \quad g_{t_{j}, \sigma_{j, t_{j}}}^{3} \quad f_{j, 3}^{3}
$$$$
h_{j, 3}^{C-1}: f_{j, 3}^{C-2} \quad g_{t_{j}, \sigma_{j, t_{j}}}^{C-1} \quad f_{j, 3}^{C-1}
$$

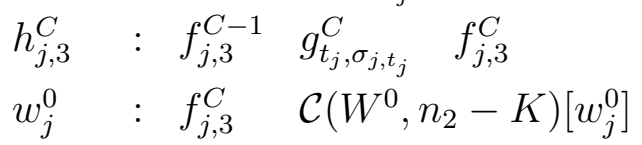

Figure 4: Preference lists of $\mathcal{W}\left(w_{j}\right)$ 